\title{
Mapas de taxas epidemiológicas: uma abordagem Bayesiana
}

\author{
Maps of epidemiological rates: \\ a Bayesian approach
}

Renato Martins Assunção 1

Sandhi M. Barreto 2

Henrique L. Guerra 2

Emília Sakurai 1

\footnotetext{
1 Departamento de Estatística, Universidade Federal de Minas Gerais. Caixa Postal 702, Belo Horizonte, MG 30161-970, Brasil. assuncao@est.ufmg.br emilia@est.ufmg.br 2 Laboratório de Epidemiologia e Antropologia Médica, Instituto René Rachou, Fundação Oswaldo Cruz. Av. Augusto de Lima 1715, Belo Horizonte, MG 30190-002, Brasil.
}

Abstract This article presents statistical methods recently devel oped for the analysis of maps of disease rates when the geographic units have small populations at risk. They adopt the Bayesian approach and use intensive computational methods for estimating risk in each area. The objective of the methods is to separate the variability of rates due to differences between regions from the background risk dueto pure random fluctuation. Risk estimates have a total mean quadratic error smaller than usual esti mates. Weapply these new methods to esti mate infant mortal ity risk in the municipalities of the State of Minas Gerais in 1994.

Key words Maps; Epidemiology; Medical Geography; Small-Area Analysis

Resumo Nesteartigo, apresentamos métodos estatísticos desen volvidos recentemente para a anál ise de mapas de taxas de morbi dade quando as unidades geográficas possuem pequenas populações de risco. El es adotam a abordagem Bayesiana e utilizam métodos computacionais intensivos para esti mação do risco de cada área. O obj etivo dos métodos é separar a variabilidade das taxas devida às di ferenças entre as regi ões do risco subjacente daquela devi da à pura flutuação aleatória. As estimativas de risco possuem um erro quadrático médio total menor que as estimativas usuais. Aplicamos esses novos métodos para estimar o risco de mortalidade infantil nos municípios de Minas Gerais em 1994.

Palavras-chave Mapas; Epidemiologia; Geografia Médica; Análise de Pequenas Áreas 


\section{Introdução}

A análise da dispersão espacial do risco de uma doença é feita principal mente através de mapas de taxas de incidência ou al guma outra medida epidemiológica de risco. Estes mapas são instrumentos valiosos em estudos epidemiológi cos e podem-se listar três objetivos principais de sua produção. O primeiro é descritivo e consiste da simples visualização da distribuição espacial da doença na região de interesse. Glass et al. (1995), por exemplo, produziram um mapa de risco de doença de Lyme a partir de dados epidemiológicos e de um sistema de informação geográfica. O segundo objetivo, exploratório, é sugerir determinantes locais de doenças e fatores etiológicos desconhecidos que possam ser formulados em termos de hi póteses a serem investigadas posteriormente. Por exemplo, Mason (1995) apresenta os vários estudos de campo realizados como conseqüência das questões ligadas aos determinantes ambientais do câncer levantadas após analise dos diversos atlas editados pelo National Cancer Institute americano. Estes estudos abrangem câncer oral (Winn et al., 1981), câncer do intestino (Pickle et al., 1981), câncer de pulmão (Ziegler et al., 1984), câncer de bexiga (Hoover \& Strasser, 1980). Finalmente, um terceiro objetivo é apontar associações entre fontes potenciais de contaminação e áreas de risco elevado, tal como aumento de risco de leucemia infantil nas proximidades de instalações nucleares (Diggle et al., 1990; Elliot et al., 1992). Neste caso, existe uma hipótese ou suspeita prévia de que a vizinhança de um ou mais pontos pré-especificados possuem risco mais elevado e trata-se então de obter evidência da existência desse efeito.

A maior parte dos mapas epidemiológicos são constituídos por mapas temáticos onde um conjunto de áreas é sombreada de acordo com seus valores para certa variável de interesse. Considere, por exemplo, o mapa da Figura 1 que mostra Minas Gerais subdividida nos seus 756 municípios existentes em 1994. De acordo com o val or da razão de mortalidade infantil padronizada (RMP ou, em inglês, SMR), os municípios receberam diferentes cores e/ ou tonalidades. Este número é igual a 100 vezes a razão entre o número observado $Y_{i}$ de óbitos em menores de um ano no município i e o seu número esperado $\mathrm{N}_{\mathrm{i}}$. r sob risco constante, onde $\mathrm{N}_{\mathrm{i}}$ é o número de nascidos vivos em 1994 no município i e r é a razão entre o número total de óbitos em menores de um ano e o número total de nascidos vivos no estado.

O mapa fornece uma descrição da distribuição da mortalidade infantil no estado e sua característica mais saliente é a forte correlação espacial entre as taxas: municípios vizinhos tendem a ter taxas mais similares do que dois municípios escol hidos ao acaso dentre os 756 existentes. Bolsões com taxas padronizadas muito elevadas ou muito baixas são facilmente percebidos no extremo nordeste e extremo noroeste. Note que os municípios com RMP iguais a zero foram deixados sem cor.

O mapa também apresenta características que revelam alguns dos seus problemas. Notase a presença de alguns municípios com valores muito diferentes de seus vizinhos. Isto cria uma aparência de colcha de retal hos que dificulta a visualização de tendências e gradientes em larga escala. Mais importante ainda, vários dos valores discrepantes e mais extremos são causados por flutuação aleatória, sem nenhuma associação com o risco subjacente. Quando o número de nascidos vivos é pequeno, a RMP pode variar enormemente devido a uma pequena mudança no número de óbitos. Considerando que a extensão de sub-registro de nascimentos e de óbitos infantis no Brasil varia muito por município, esse comportamento da RMP causa preocupação.

Esse grau de variabilidade al eatória da RMP está associado ao tamanho das unidades geográficas de análise. Por um lado, para alcançar plenamente seus objetivos, os mapas devem possuir resolução geográfica adequada. Isto implica que a maior utilidade dos mapas ocorre quando eles utilizam pequenas regiões geográficas como unidades de análise. Por outro lado, várias dessas pequenas regiões possuirão pequenas populações de risco o que acarretará estimativas de risco muito instáveis. Isto é, o acréscimo ou decréscimo de um único caso nestas áreas poderá causar mudanças drásticas nas estimativas. Em termos estatísticos, as estimativas das diversas áreas não são comparáveis já que possuem variâncias muito diferentes. A dependência da variância de RMP no tamanho da população de risco é vista claramente na Figura 2 que mostra um gráfico de dispersão de RMP versus o logaritmo do número de nascidos vivos para os municípios de $\mathrm{Mi}$ nas Gerais em 1994. Quanto menor o tamanho da população de risco, maior a variabilidade da RMP.

Flutuações extremas nas taxas dissociadas dos riscos subjacentes levam, al gumas vezes, à decisão de não divulgá-las quando as áreas são muito pequenas como, por exemplo, a nível municipal. Uma alternativa é então perder resolução fazendo um mapa subdividido em áreas de análise maiores por meio da agregação das áreas menores. No entanto, isto entra 
em conflito com um dos principais objetivos de se fazer os mapas que seria a identificação das áreas de maior risco para orientar intervenções de saúde pública. É fundamental alocar recursos de forma não-agregada, respeitando as diferenças e necessidades locais, que só podem ser avaliadas quando as áreas de análise são pequenas o suficiente. As flutuações aleatórias podem ser ainda maiores em estudos de mortalidade segundo causa específica, essencial para a monitorização de ações epidemiológicas e de saúde pública no nível local.

Num artigo clássico e pioneiro, Choynowski (1959) considera a ocorrência de tumores cerebrais em condados poloneses. Ele nota que os dois casos observados em Lesko com população de 17000 habitantes gera uma taxa relativamente extrema de 11,8 por 100 mil. Se tivesse ocorrido apenas um caso, ao invés dos dois observados, essa taxa seria de 5,9, um vaIor consistente com as taxas dos outros condados. Este problema também está presente em nossos dados. Existem 15 municípios com nenhum óbito registrado e com número de nascidos vivos inferior a 30 implicando uma RMP igual a zero. Se uma única morte houvesse sido registrada nesses municípios, as razões de mortalidade padronizada passariam de 0 para valores entre 116 e 1048. O último valor corresponderia a um município com risco relativo estimado 10 vezes maior que a média do estado e passaria então a ser o mais alto substituindo o valor extremo de fato observado que foi 609 . Teríamos assim os aspectos mais salientes do mapa causados pela sensibilidade extrema da RMP a pequenas mudanças no numerador, possivelmente devidas a flutuações aleatórias não associadas ao verdadeirro risco subjacente.

Para evitar esse efeito drástico de mudanças mínimas no número de óbitos registrados, Choynowski (1959) propõe fazer mapas substituindo as taxas por probabilidades similares ao p-valor de um teste. Um mapa temático baseado nessa proposta é chamado de mapa de probabilidade. Embora a idéia deste mapa seja permitir comparações por meio da padronização das taxas em uma escala de probabilidade, isto não é possível se algumas populações de risco são grandes pois então teremos valores extremos da probabilidade como conseqüência do poder do teste para detectar pequenos afastamentos da hipótese de risco constante utilizada para o cálculo das probabilidades (Cressie, 1991).

Existem outras desvantagens na utilização desses mapas de probabilidade. Os p-valores não possuem uma interpretação epidemiológica clara. Eles também não levam em conta as esperadas similaridades entre áreas contíguas. Estas similaridades são devidas à usual variação suave do risco sobre a área sendo mapeada. Incorporar esta informação nas estimativas de risco pode levar a mapas com menos flutuação al eatória e assim a uma diferenciação mais precisa entre o que é de fato risco el evado e o que é flutuação aleatória causada por pequenas populações ou grande potência de detectar diferenças substantivamente desprezíveis.

Para superar estas dificuldades, métodos Bayesianos empíricos ou inteiramente Bayesianos têm sido propostos na literatura. Estes métodos, ao estimar o risco de uma pequena área, têm como idéia central o uso de informação das outras áreas que compõem a região de estudo para diminuir o efeito das flutuações aleatórias não associadas ao risco. Ao levarem em conta a correlação espacial entre áreas vizinhas, os mapas resultantes são mais suaves e mais informativos (Mason et al., 1975; Mason et al., 1976). A principal conseqüência é que o conjunto dos riscos relativos de todas as áreas é estimado de forma mais precisa do que ao usar a RMP. Isto é, pode-se diminuir substancialmente o erro quadrático médio total da estimação dos riscos. Os ganhos em termos do decréscimo do erro quadrático de estimação podem ser substanciais.

Os primei ros avanços estatísticos usaram a abordagem Bayesiana empírica (Tsutakawa, 1988; Tsutakawa et al., 1985; Manton et al., 1989). Em 1987, Clayton \& Kaldor propuseram incluir uma especificação a priori espacial para levar em conta a correlação entre áreas vizinhas. Em seguida, Clayton (1989) propôs uma abordagem Bayesiana hierárquica que não possui as desvantagens da RMP e mostra algumas vantagens comparadas com as al ternativas Bayesianas empíricas (Bernadinelli \& Montonolli, 1992).

Mais recentemente, Besag et al. (1991) propuseram uma abordagem em que os dados observados são o produto de dois estágios. No primeiro, o risco relativo de cada área é a soma de dois componentes representando fatores espaciais e não-espaciais. No segundo, dado este risco a priori e as populações de risco, a contagem da característica de interesse em cada área tem distribuição Poisson com média especificável. As distribuições a priori para os componentes do primeiro estágio podem ser escolhidas de modo a refletir o conhecimento epidemiológico sobre a doença na área, incluindo com diferentes escopos, as variáveis 


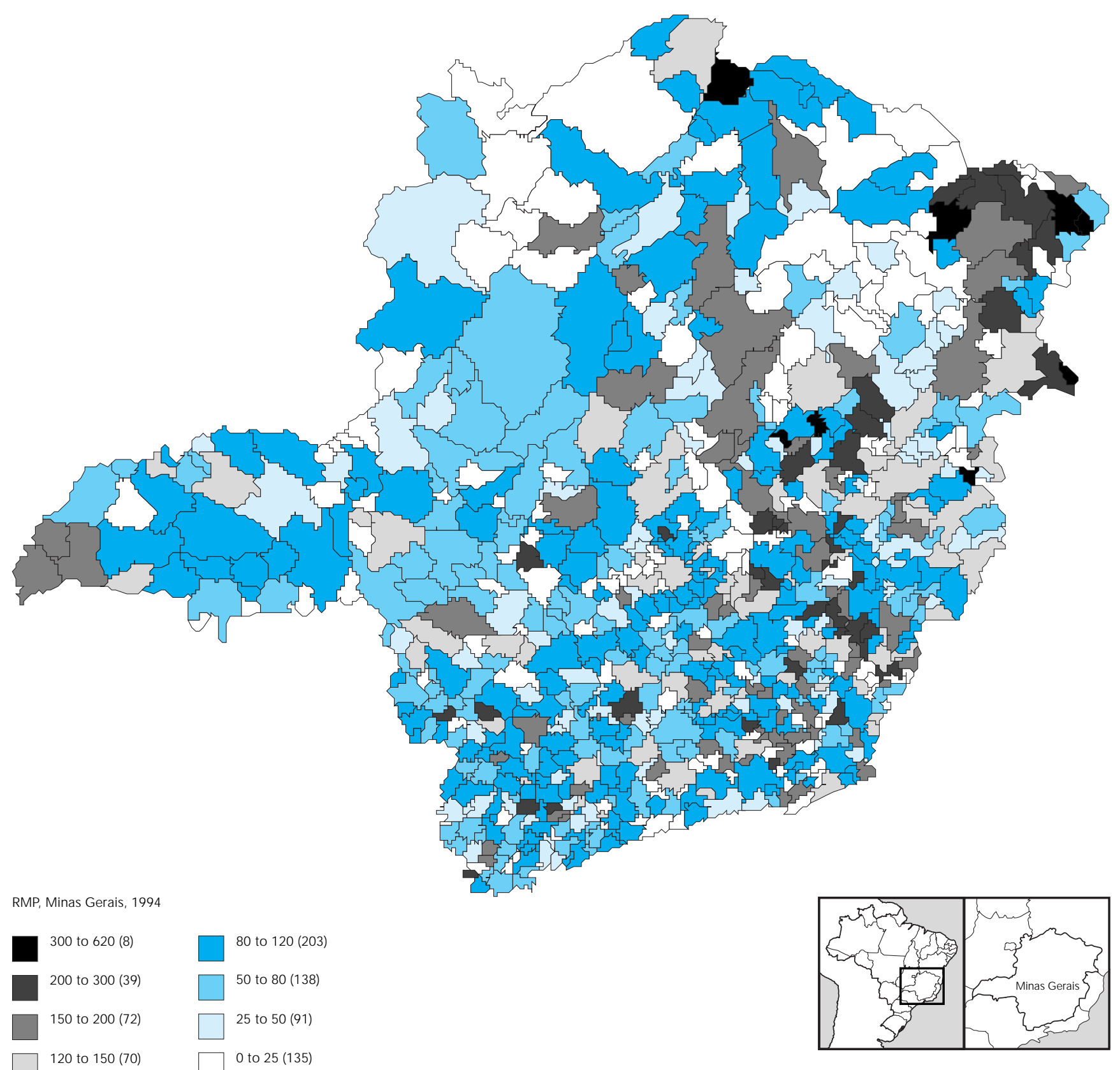

não-observadas, como em Bernadinelli et al. (1995a; 1995b). A correlação espacial seria exibida apenas por aquelas variáveis com variabilidade de larga escala relativa, enquanto que aquelas com variação de pequena escala seriam mais restritas às pequenas áreas sob consideração e não teriam estrutura espacial.

\section{Modelo hierárquico Bayesiano}

Nesta seção, nós introduzimos concisamente o modelo Bayesiano hierárquico espacial. Uma revisão detalhada foi apresentada por Bernadinelli \& Montomoli (1992). A estrutura estatística de nosso problema, considerando um estu- 


\section{Figura 2}

Diagrama de dispersão do logaritmo do número de nascidos vivos no município (eixo horizontal) versus RMP (eixo vertical).

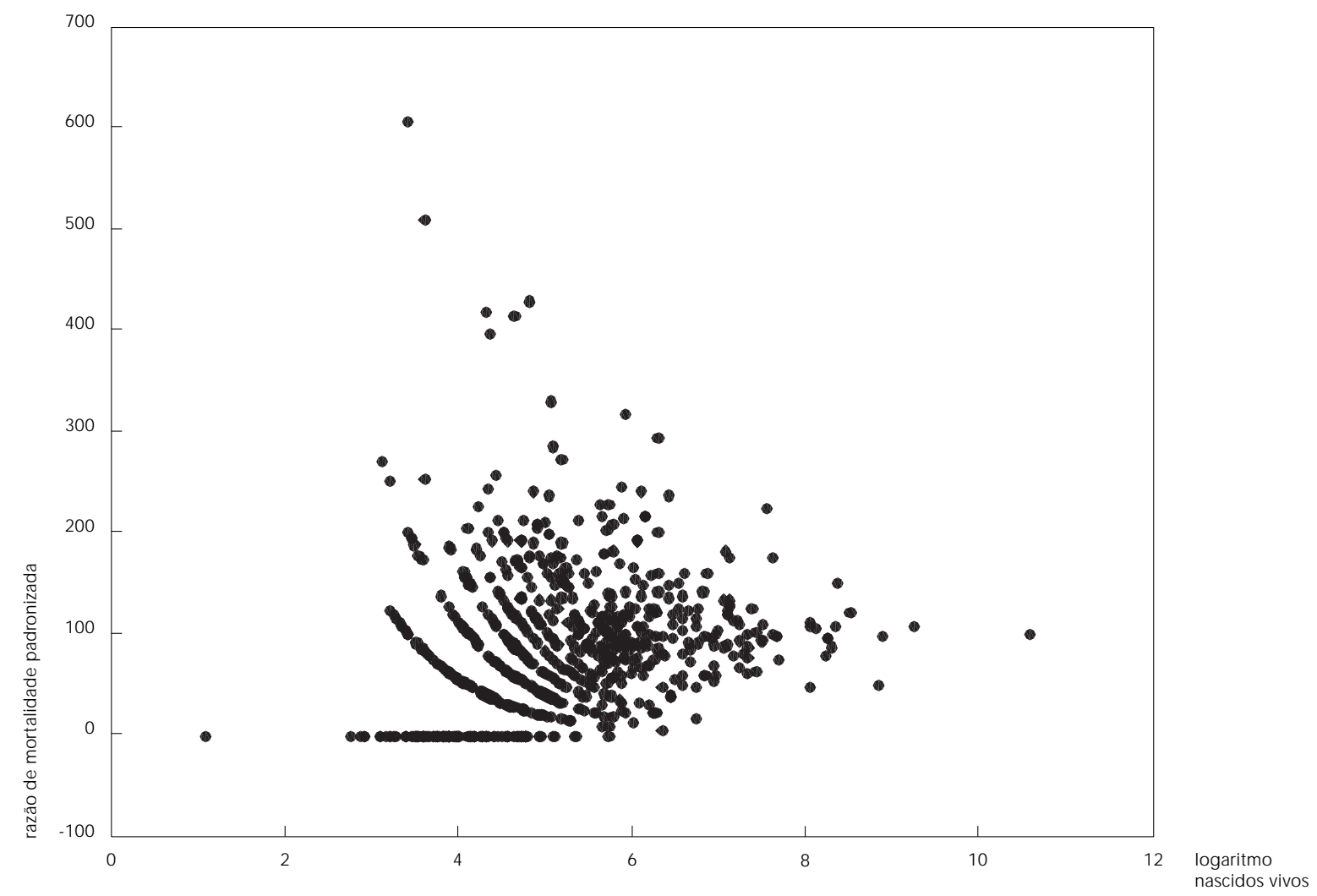

do de mortalidade para n municípios, indexados por $\mathrm{i}=1, \ldots, \mathrm{n}$, pode ser descrita a partir de três informações: dos riscos relativos $\psi_{i}$, que expressam as difrenças relativas entre os muncípios; dos valores observados de óbitos $Y_{1}, \ldots, Y_{n}$ e dos esperados de óbitos $E_{1}, \ldots, E_{n}$ obtidos aplicando-se taxas de referência apropriadas à população local, sob a hipótese de que, em cada categoria de idade, o risco é constante para todas os municípios. Desse modo, no município i, o número de mortes esperado é dados $\mathrm{E}_{i} \psi_{\mathrm{i}}$. Assim, parte da variação das observações é devida a uma flutuação aleatória em cima dos riscos subjacentes. Ainda mais, podese dizer que, de posse dos valores $\mathrm{E}_{i}$ e de $\psi_{i}$, o número de óbitos no município i segue uma distribuição de Poisson com média igual a $E_{i} \psi_{i}$.

O risco relativo $\psi_{i}$ é o produto de dois componentes independentes: o primeiro registra o efeito aleatório não-espacial $\left(\theta_{\mathrm{i}}\right)$, enquanto que o segundo é o efeito aleatório espacial do município i $\left(\phi_{\mathrm{i}}\right)$.
Os elementos $\theta_{i}$ do primeiro componente possuem distribuições normal independentes com média 0 e variância $1 / \tau_{\theta}$. A distribuição de probabilidades do segundo componente é definida de modo a incentivar áreas vizinhas a serem mais semel hantes em termos de risco de mortalidade do que duas áreas escolhidas ao acaso. Na linguagem estatística, diz-se que para um município i, a distribuição de $\phi_{\mathrm{i}}$, em função do restante do conjunto dos $\phi_{k}, k \neq i$, é definida por um campo de Markov, em que a distribuição condicional desse segundo componente depende apenas de sua vizinhança $\partial_{i}$. Isto implica que $\phi_{i}$ possui distribuição normal com média $m_{\partial i}$ e variância $\sigma^{2}{ }_{\partial i}$ onde $m_{\partial i}$ éa média dos $\phi_{k}$ vizinhos de i e $\sigma z_{\partial i}$ é inversamente proporcional ao número de vizinhos do lote i.

Os hiperparâmetros $\tau_{\theta}$ e $\tau_{\phi}$ possuem uma distribuição a priori, a escolha comum recaindo sob uma distribuição qui-quadrado ou uma distribuição gama. Neste artigo, nós seguimos Bernadinelli \& Montomoli (1992) e estabelece- 
mos que $\tau_{\phi}$ possui distribuição igual a a $\cdot \chi_{v}^{2} / v e$ $\tau_{\theta}$ possui distribuição $b \cdot \chi_{u}^{2} / u$ onde $a$ e b são duas constantes especificadas representando estimativas a priori de $\tau_{\theta}$ e $\tau_{\phi}$. Essas constantes são escolhidas, por exemplo, supondo que os riscos relativos das diversas áreas devem variar entre, digamos, 1/ 3 e 3 para a maioria dos municípios (Bernadinelli e Montomoli, 1992). Os graus de liberdade $u$ e $v$ representam o grau de confiança nessas estimativas a priori.

A inferência Bayesiana segue da análise da densidade a posteriori conjunta dos dois parâmetros referentes à estrutura espacial e nãoespacial $\left(\phi_{1}, \ldots, \phi_{\mathrm{n}}, \theta_{1}, \ldots, \theta_{\mathrm{n}}, \tau_{\phi}, \tau_{\theta}\right)$. Um método de se obter essa distribuição conjunta é através da simulação de um número suficientemente grande de observações dependentes do vetor de parâmetros como uma cadeia de Markov ergódica (Besag et al., 1991; Besag \& Green, 1993). Esse método é conhecido como Amostrador de Gibbs e pode ser implementado através do uso do software BUGS, disponível gratuitamente na internet no endereço http:// www.mrc-bsu.cam.ac.uk/ bugs. A distribuição assintótica desta cadeia é a distribuição conjunta a posteriori do vetor de parâmetros e assim, se a cadeia é longa o suficiente, observações podem ser obtidas para construir uma função de distribuição empírica a partir da qual pode-se estudar as propriedades da distribuição a posteriori do vetor de parâmetros. Por exemplo, a estimativa pontual para o risco relativo é a média a posteriori da distribuição de $\psi_{i}$.

\section{Resultados}

Para implementar a metodologia, definimos uma estrutura de vizinhança simples. Alguns municípios tiveram como vizinhos todos aqueles que tinham suas sedes a um raio menor que 5 unidades na escala do mapa enquanto para outros foi adotado o raio de 10. Os municípios com raio menor são aproximadamente aqueles da metade inferior do mapa onde as sedes estão muito próximas. O raio maior foi adotado para aqueles que tinham áreas maiores e são esparsamente povoados como os do norte de Minas. Os graus de liberdade das distribuições dos hiperparâmetros foram iguais a 1 e as constantes daquelas distribuições foram escoIhidas de modo a permitir uma variação relativa a priori dos riscos de até 4 vezes um valor médio. Simulações com outras escolhas dos parâmetros não apresentam mudanças significativas nos resultados.

A Figura 3 mostra o resultado da aplicação da metodologia proposta aos municípios mi- neiros. Os eixos vertical e horizontal possuem a mesma escala e a linha sólida no gráfico é a reta $y=x$. As estimativas Bayesianas do risco relativo são muito mais concentradas do que as estimativas fornecidas pelas RM P. O efeito desta contração das estimativas é maior nos valores extremos de RMP. Valores acima de 300 foram reduzidos enquanto valores próximos ou abaixo de zero foram puxados para perto da média da distribuição. O efeito da contração depende da população em risco de cada município. Aqueles com grande população não tiveram grandes mudanças em relação à RMP já que estas devem ser estimativas confiáveis do risco subjacente. Já aqueles com pequena população de risco usaram também a informação vinda dos municípios vizinhos já que a RMP seria instável.

O efeito visual das estimativas Bayesianas pode ser observado no mapa da Figura 4.0 mapa possui uma aparência muito mais suave e demonstra mais claramente os padrões espaciais do risco de mortalidade infantil. Os bolsões de riscos relativos semelhantes são nítidos e facilmente identificáveis. Além disto, as estimativas municipais podem ser analisadas com mais segurança pois são estimadas com maior estabilidade. Os valores discrepantes resultantes podem ser estudados sem o temor de que sejam resultado de flutuação aleatória causada por populações pequenas. Nota-se, por exemplo, um município, Caetanópolis, na região central do estado com uma estimativa acima de 200 cercado por municípios com riscos relativos entre 100 e 150 . A região do noroeste minei ro apresenta riscos relativos baixos e é muito homogênea. A partir do sul de Minas, passando pela Zona da Mata, Vale do Rio Doce e Zona Metalúrgica até o Médio Jequitinhonha no extremo nordeste podemos enxergar um gradiente de aumento do risco que é quebrado pelo bolsão de risco relativo baixo na região do Alto Jequitinhonha. Esta descontinuidade no gradiente pode ser explicada pelo sub-registro de óbito. Análises específicas e localizadas incluindo estudos de tendências temporais em pequenas áreas podem ser realizadas com mapas produzidos com esta metodologia.

\section{Simulação}

Como demonstrar que a metodologia proposta neste artigo é melhor que o uso das tradicionais RMP? Uma prova definitiva seria através da comparação entre os erros médios da estimação dos riscos feita pelos dois métodos. No entanto, isto é impossível já que os riscos não 


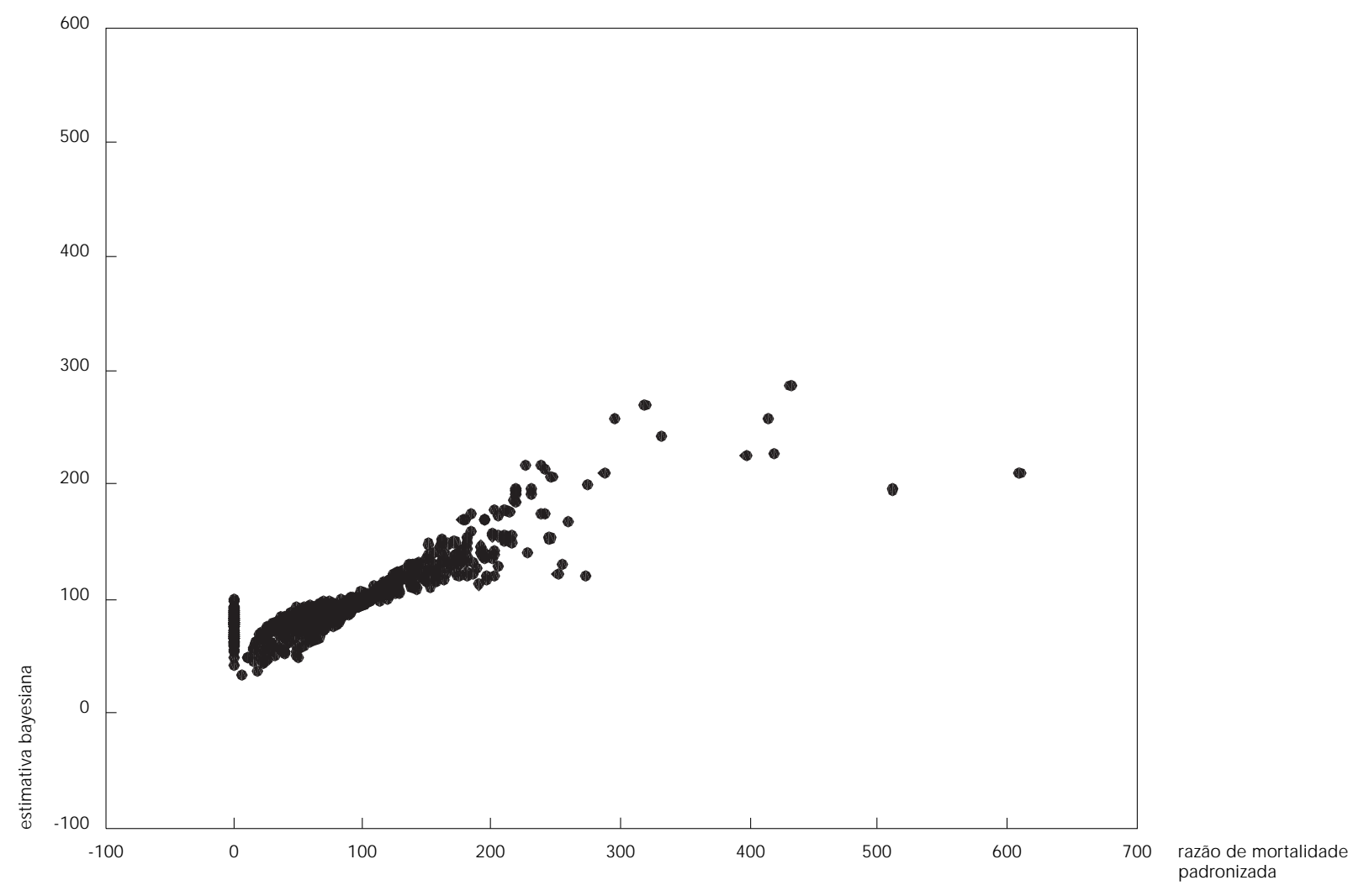

podem ser observados diretamente. No contexto de modelos probabilísticos é possível mostrar que as estimativas Bayesianas são meIhores que a RMP (Lehman, 1983). No entanto, para argumentar a favor de nossa proposta, utilizamos um método de simulação que nos parece simples e convincente. Tomamos o risco relativo estimado pelo método Bayesiano como se fosse o risco verdadeiro, não observado, e aplicamos este risco nos nascidos vivos de cada município para obter o risco de óbito infantil no município. A seguir, simulamos o número de mortes em cada município de acordo com a distribuição de Poisson. Tomando estes novos dados, ajustamos o modelo Bayesiano e obtivemos os riscos relativos estimados pelo método Bayesiano e pelas RMP.

A comparação entre esses dois conjuntos de estimativas é feita através da Figura 5 e calculando-se o erro médio quadrático de estimação de cada um. Este erro médio é definido como EQM $=\Sigma_{i}\left(\psi_{i}-\hat{\psi}_{i}\right) 2$, onde o elemento com chapéu é a estimativa do risco relativo. $\mathrm{Na} \mathrm{Fi-}$ gura 5 fica claro que as estimativas Bayesianas são muito estáveis e precisas na estimação do risco relativo. A diferença entre os métodos e o erro de estimação de RMP é maior quando o número de nascidos vivos é pequeno. O erro médio quadrático do estimador Bayesiano é 73,9 enquanto o das razões RMP é igual a 2116,7 , uma diferença relativa de $97 \%$ em relação a este último. Trocando o critério de medição do erro médio não muda muito os resultados. Por exemplo, ao invés do erro quadrático, o erro absoluto relativo médio, em termos percentuais, do estimador Bayesiano é $6 \%$ enquanto o das taxas RMP é igual a 34\%. Como se vê, a diferença é bastante significativa em favor das estimativas Bayesianas.

\section{Conclusão}

Neste artigo, utilizamos um método Bayesiano para estimar o risco relativo de mortalidade infantil nos municípios de Minas Gerais usando 


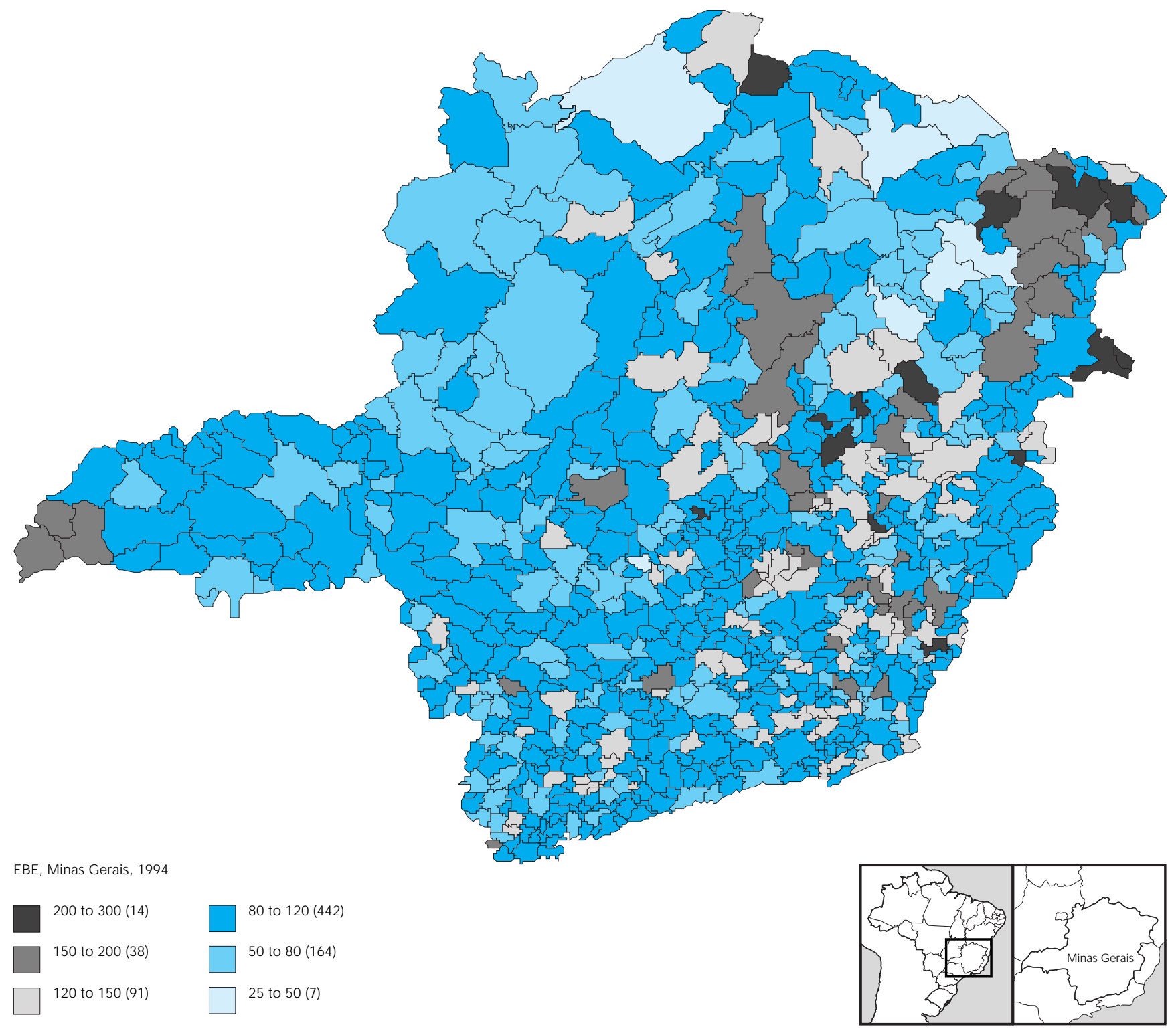

os dados de 1994. O método é intensivo computacionalmente e utiliza métodos Monte Carlo via Cadeias de Markov para estimar a distribuição a posteriori dos riscos relativos. Além disto, o modelo é intrisicamente espacial incorporando um componente que capta a variação suave de larga escala do risco no estado. $O$ resultado é o decréscimo da influência de fatores aleatórios não relacionados com o risco subjacente e a produção de mapas mais confiáveis e fáceis de interpretar.
É importante salientar que este método não afeta erros sistemáticos como aqueles causados por sub-registro e isto implica que a interpretação dos mapas requer um conhecimento sobre a qualidade dos dados e as áreas estudadas. Note, por exemplo, que o sub-registro de óbitos parece ser a explicação mais plausível para o bolsão de baixo risco estimado no mapa Bayesiano no Alto Jequitinhonha e também no Norte de Minas, já que estas áreas estão entre as mais pobres de Minas Gerais. $\mathrm{O}$ 


\section{Figura 5}

Resultados referentes à simulação. 0 primeiro gráfico é do risco relativo versus a estimativa Bayesiana.

0 segundo gráfico é de RMP versus a estimativa Bayesiana e o terceiro é de RMP versus o risco relativo.

0 quarto gráfico é logaritmo de nascidos vivos versus o erro de estimação usando RM P.

$5 a$

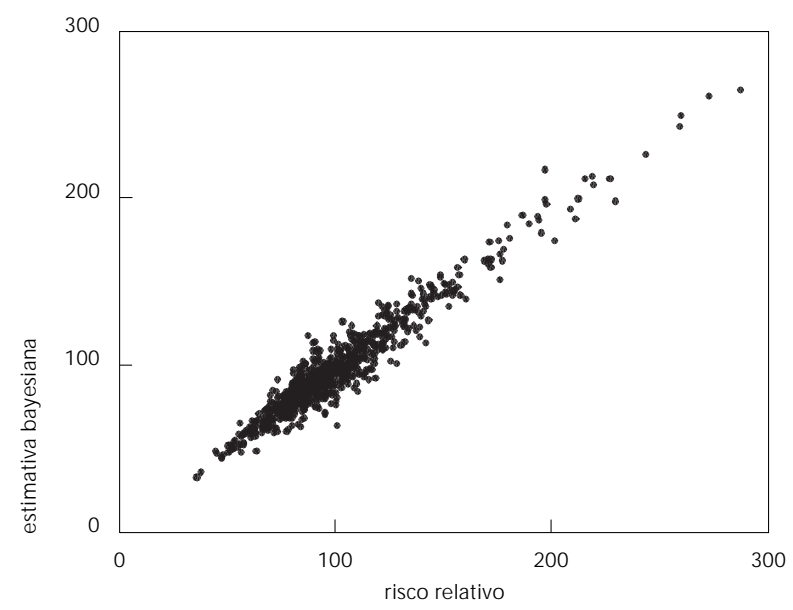

$5 c$

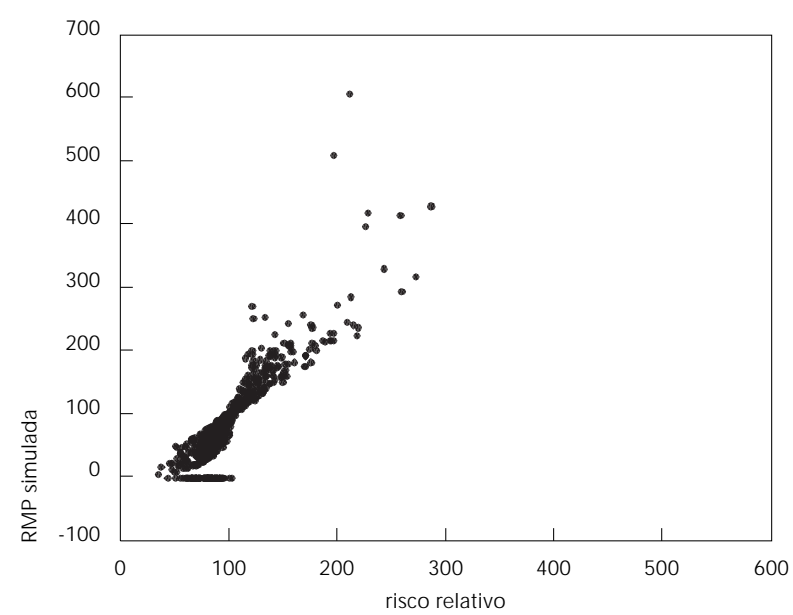

$5 b$

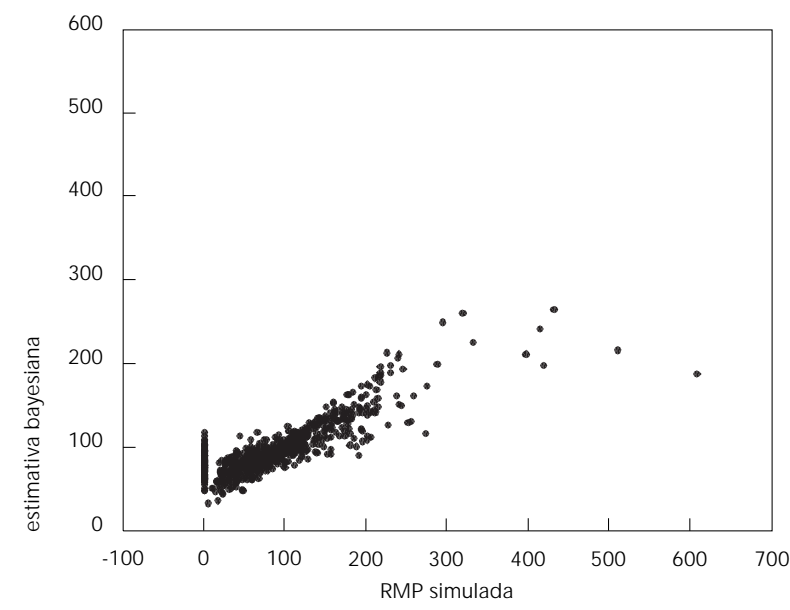

$5 d$

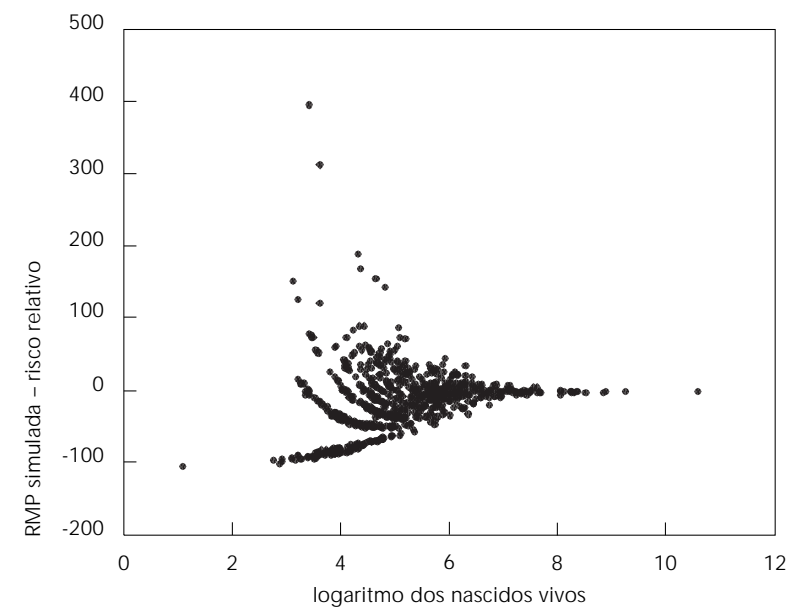

sub-registro de óbitos e nascimentos tende a ser maior exatamente nessas áreas menos desenvolvidas, compostas de municípios pouco populosos e predominantemente rurais. $\mathrm{O}$ sub-registro de nascimentos teria um impacto maior nos municípios menos populosos, embora este efeito tenda a produzir oscilações menores que o sub-registro de óbitos. Primeiro, porque ele afetaria o denominador dessas taxas que é um número muito maior que o de óbitos. Segundo, porque o registro de nascimentos tende a crescer com o tempo de sobrevida da criança, ou seja, haveria um maior subregistro de crianças que faleceram precocemente.

Existe um grande campo para explorar meIhorias no método. Uma das características mais marcantes do método é o campo de Mar- 
kov que permite correlação entre as áreas. Este campo é definido parcialmente pela estrutura de vizinhança adotada. Existem várias possibilidades para essa estrutura de vizinhança e o efeito de mudanças de vizinhanças nas estimativas não foi estudado na literatura. Por exemplo, pode-se definir como vizinhos dois municípios que possuam fronteira comum. Outra al ternativa é considerar como vizinhos de um município todos aqueles que estejam num raio de x quilômetros de sua sede. Nas duas alternativas acima, teremos um número variável de vizinhos. Outra possibilidade que mantém um número fixo de vizinhos é tomar apenas aqueles municípios que correspondem às $k$ sedes mais próximas do município base. Nós planejamos estudar uma estrutura diferente onde o número de vizinhos é variável mantendo, no entanto, a população de risco destes municípios aproximadamente constante. Nós toma-

\section{Agradecimentos}

Desejamos agradecer ao Cedeplar-UFM G e ao Office of Population Research-Princeton University pelo excelente ambiente de trabal ho que proporcionaram ao primeiro autor durante a real ização deste artigo e a Edna A. Reis pela ajuda com o texto. Foram muito benéficas as discussões mantidas com Diana Sawyer e equipe, German Rodrigues, Burton Singer e Max Woodbury. Este trabalho contou com o apoio da Fundação Rockfeller, IDRC-Canadá, Fapemig e CNPq. remos como vizinhos aqueles mais próximos até que se ultrapasse um ponto de corte na população de risco acumulada. Isto equaliza o número de casos utilizados para estimar cada risco.

Várias áreas da epidemiologia podem se beneficiar do método proposto aqui. Uma delas é a correção de sub-registro de pequenas áreas onde a incerteza nas estimativas das taxas demográficas devido à pequena população das áreas leva a grande erro relativo. Em geral, os métodos atual mente empregados não consideram a estrutura espacial subjacente no processo demográfico. Parece ser possível fazer uma extensão do método exposto aqui para esse problema e este é um dos problemas com o qual nos ocuparemos. Em geral, qualquer problema onde taxas são calculadas para muitas áreas contíguas e com populações pequenas poderia se beneficiar desta metodologia.

\section{Referências}

ANDES, N. \& DAVIS, J. E., 1995. Linking public data using geographical information systems techniques: alaskan community characteristics and infant mortality. Statistics in Medicine, 14:481490.

BERNADINELLI, L. \& MONTONOLLI, C., 1992. Empirical bayes versus fully bayesian analysis of geographical variation in disease risk. Statistics in Medicine, 11:983-1007.

BERNADINELLI, L.; CLAYTON, D. \& MONTOMOLI, C., 1995a. Bayesian estimates of disease maps: how important are priors? Statistics in Medicine, 14:2411-2431.

BERNADINELLI, L.; CLAYTON, D.; PASCUTTO, C.; MONTONOLLI, C.; GHISLANDI, M. \& SONGINI, M., 1995b. Bayesian analysis of space-time variation in disease risk. Statistics in Medicine, 14: 2433-2443. 
BESAG, J. \& GREEN, P. J., 1993. Spatial Statistics and Bayesian computation. Journal of the Royal Statistical Society, 55:25-37.

BESAG, J.; YORK, J. \& MOLLIÉ, A., 1991. Bayesian image restoration, with applications in spatial statistics (with discussion). Annals of the Institute of Statistical Mathematics, 43:1-59.

CHOYNOWSKI, M., 1959. Maps based on probabilities. Journal of the American Statistical Association, 54:385-388.

CLAYTON, D. 1989. Hierarchical Bayesian models in descriptive epidemiology. Proceedings of theXIVth International Biometrics Conference, 201-213.

CLAYTON, D. \& KALDOR, J., 1987. Empirical Bayes estimates of age-standardized relative risks for use in disease mapping. Biometrics, 43:671-681.

CRESSIE, N., 1991. Statistics for Spatial Data. Chichester: John Wiley \& Sons.

GLASS, G. E.; SCHWARTZ, B. S.; MORGAN, J. M.; JOHNSON, D. T.; NOY, P. M . \& ISRAEL, E., 1995. Environmental risk factors for Lyme disease identified with geographic information systems. American Journal of Public Health, 85:944-948.

DIGGLE, P. J.; GATRELL, A. C. \& LOVETT, A. A., 1990. Modelling the prevalence of cancer of cancer of larynx in part of Lancashire: a new methodology for spatial epidemiology. In: Spatial Epidemiology (R. W. Thomas, ed.), pp. 153-171. Pion: London.

ELLIOTT, P. J.; HILLS, M.; BERESFORD, J.; KLEINSCHMIDT, I.; JOLLEY, D.; PATTENDEN, S.; RODRIGUES, L.; WESTLAKE, A. \& ROSE, G., 1992. Incidence of cancer of the larynx and lung cancer near incinerators of waste solventes and oil in Great Britain. The Lancet, 339:854-858.

HOOVER, R. N. \& STRASSER, P. H., 1980. Artificial sweeteners and human bladder cancer: preliminary results. Lancet, 1:837-840.

LEHMAN, E. L., 1983. Theory of Point Estimation. New York: John Wiley \& Sons.
MANTON, K. G.; WOODBURY, M. A.; STALLARD, E.; RIGGAN, W. B.; CREASON, J. P. \& PELLOM, A. C., 1989. Empirical bayes procedures for stabilizing maps of U.S. cancer mortality rates. Journal of the American Statistical Association, 84:637-650.

MASON, T. J., 1995. The development of the series of U.S. cancer atlases: implications for the future epidemilogic research. Statistics in Medicine, 14: 473-479.

MASON, T. J.; MCKAY, F. W.; HOOVER, R.; BLOT, W. J. \& FRAUMENI Jr., J. F., 1975. Atlas of Cancer Mortality for U.S. Counties, 1950-1969. Washington: Department of Health, Education, and Welfare Publication 75-780, U.S. Government Printing Office.

MASON, T. J.; MCKAY, F. W.; HOOVER, R.; BLOT, W. J. \& FRAUM ENI Jr., J. F., 1976. Atlas of Cancer Mortality among U.S. Nonwhites: 1950-1969. Washington: Department of Health, Education, and Welfare Publication 76-1204, U.S. Government Printing Office.

PICKLE, L. W.; GREENE, M. H.; ZIEGLER, R. G.; TOLEDO, A.; HOOVER, R.; LYNCH, H. T. \& FRAUMENI Jr., J. F, 1981. Colorectal cancer in rural Nebraska. Cancer Research, 44:363-369.

TSUTAKAWA, R. K., 1988. Mixed model for analysing geographic variation in mortality rates. Journal of the American Statistical Association, 83:37-42.

TSUTAKAWA, R. K.; SHOOP, G. L. \& MARIENFELD, C. J., 1985. Empirical bayes estimation of cancer mortality rates. Statistics in Medicine, 4:201-212.

WINN, D. M.; BLOT, W. J.; SHY, C. M.; PICKLE, L. W.; TOLEDO, A. \& FRAUMENI J r., J. F., 1981. Snuff dipping and oral cancer among women in the southern United States. New England Journal of Medicine, 304:745-749.

ZIEGLER, R. G.; MASON, T. J.; STEMHAGEN, A.; HOOVER, R.; SCHOENBERG, J. B.; VIRGO, P.; WALTM AN, R. \& FRAUM ENI Jr., J. F., 1984. Dietary carotene and vitamin $A$ and risk of lung cancer among white men in New Jersey. Journal of the National Cancer Institute, 73:1429-1435. 